\title{
The Foldy-Wouthuysen Transformation of the Dirac Equation in Noncommutative Phase-Space
}

\author{
Ilyas Haouam, Lyazid Chetouani \\ Département de Physique, Faculté des sciences exactes, Université des Frères Mentouri, Constantine, Algeria \\ Email: ilyashaouam@live.fr
}

How to cite this paper: Haouam, I. and Chetouani, L. (2018) The Foldy-Wouthuysen Transformation of the Dirac Equation in Noncommutative Phase-Space. Journal of Modern Physics, 9, 2021-2034.

https://doi.org/10.4236/jmp.2018.911127

Received: November 9, 2015

Accepted: September 11, 2018

Published: September 14, 2018

Copyright (C) 2018 by authors and Scientific Research Publishing Inc. This work is licensed under the Creative Commons Attribution International License (CC BY 4.0).

http://creativecommons.org/licenses/by/4.0/

\begin{abstract}
A method of Foldy-Wouthuysen transformation for relativistic spin-1/2 particles in external fields is proposed; in the present work the basic properties of the Dirac hamiltonian in the FW representation in the noncommutative phase-space are investigated and the Schrödinger-Pauli equation is found, knowing that the used methods for extracting the full phase-space noncommutative Dirac equation are, the Bopp-shift linear translation method, and the Moyal-Weyl product (*-product).
\end{abstract}

\section{Keywords}

Foldy-Wouthuysen Transformation, Nonrelativistic Limit, Noncommutative Schrödinger-Pauli Equation, Phase-Space Noncommutativity, Noncommutative Dirac Equation, Moyal Product, Bopp-Shift Translation

\section{Introduction}

The Foldy-Wouthuysen transformation (FW) [1] is one of several methods used to investigate the low-energy limit of the relativistic Dirac equation (low-speed); due to a series of sequential unitary transformations [2], it has proven to be the favorite method to meaningfully obtain the nonrelativistic limit of the Dirac equation in which it gives the Schrödinger-Pauli equation [3] [4] [5]; in the FW representation for relativistic particles in external fields, the operators have the same form as in the nonrelativistic quantum theory, this is mainly because of the fact of the hamiltonian and all operators in this representation are block-diagonal, furthermore the basic characteristics of the FW representation are obviously described in [6] [7] [8] [9]. 
In the present work, the basic properties of the Dirac hamiltonian in the FW representation in the noncommutative phase-space are investigated and the Schrödinger-Pauli equation is found, where the common methods for extracting the full phase-space noncommutative Dirac equation are both of the linear translation method, which known as Bopp-Shift translation in which it matches between the commutative quantum mechanics and the noncommutative quantum mechanics (NCQM) [10], and the Moyal-Weyl product (*-product) [11] [12] [13] [14].

\section{Phase-Space Noncommutativity}

In the two-dimensional commutative phase-space, the coordinates $x_{i}$ and the kinetic momentum $p_{i}$ satisfy the usual canonical commutation relations

$$
\left[x_{i}, x_{j}\right]=0,\left[p_{i}, p_{j}\right]=0,\left[x_{i}, p_{j}\right]=i \delta_{i j}(i, j=1,2) .
$$

In the recent study results on the phase-space noncommutativity (PSNC) is shown that at very tiny scales (string scales) the space may not commute anymore, let us consider the operators of coordinates and kinetic momentum in a two-dimensional noncommutative phase-space $\hat{x}_{i}$ and $\hat{p}_{i}$ respectively, where the noncommutative phase-space operators satisfy the commutation relations

$$
\left[\hat{x}_{i}, \hat{x}_{j}\right]=i \Theta_{i j},\left[\hat{p}_{i}, \hat{p}_{j}\right]=i \eta_{i j},\left[\hat{x}_{i}, \hat{p}_{j}\right]=i \hbar^{e f f} \delta_{i j}(i, j=1,2),
$$

with the effective Plank constant being

$$
\hbar^{e f f}=\hbar\left(1+\frac{\Theta \cdot \eta}{4 \hbar^{2}}\right)
$$

where

$$
\Theta_{i j}=\epsilon_{i j k} \Theta_{k}, \Theta_{k}=(0,0, \Theta), \eta_{i j}=\epsilon_{i j k} \eta_{k}, \eta_{k}=(0,0, \eta),
$$

$\Theta, \eta$ are noncommutative parameters, they are real-valued and antisymmetric constant matrices with dimension of (length) ${ }^{2}$ and (momentum) ${ }^{2}$, respectively.

The noncommutativity in phase-space can be realized in terms of Moyal-Weyl product (*-product) [15] [16] [17] which means that the noncommutativity information is encoded in the Moyal product, defined as

$$
\begin{aligned}
& (f \star g)(\hat{x})=\exp \left[\frac{i}{2} \Theta_{a b} \partial_{x_{a}} \partial_{x_{b}}\right] f\left(x_{a}\right) g\left(x_{b}\right) \\
& =f(x) g(x)+\sum_{a=1}\left(\frac{1}{n !}\right)\left(\frac{i}{2}\right)^{2} \Theta^{a_{1} b_{1}} \ldots \Theta^{a_{n} b_{n}} \partial_{a_{1}} \cdots \partial_{a_{k}} f(x) \partial_{b_{1}} \ldots \partial_{b_{k}} g(x) .
\end{aligned}
$$

The noncommutative phase-space operators are related to the commutative phase-space one, due to the so-called Bopp-shift linear transformation [18] [19] [20], knowing that the latter induced from the ${ }^{\star}$-product, and it is given by

$$
\begin{array}{ll}
\hat{x}=x-\frac{1}{2 \hbar} \Theta p_{y} & \hat{y}=y+\frac{1}{2 \hbar} \Theta p_{x} \\
\hat{p}_{x}=p_{x}+\frac{1}{2 \hbar} \eta y & \hat{p}_{y}=p_{y}-\frac{1}{2 \hbar} \eta x
\end{array}
$$


If $\Theta=\eta=0$ the noncommutative phase-space algebra reduces to the commutative one.

\section{Nonrelativistic Limit of the Dirac Equation in Noncommutative Phase-Space}

\subsection{The Dirac Equation in Noncommutative Phase-Space}

As it known by the use of the ${ }^{*}$-product, we obtain the Dirac equation for the noncommutative quantum mechanics [21] [22]

$$
H(\hat{x}, \hat{p}) \star \psi(\hat{x})=E \psi,
$$

knowing that the Dirac equation in interaction with the electromagnetic four-potential $A_{\mu}$ in commutative phase-space is

$$
\left[c \alpha_{i}\left(p_{i}-\frac{e}{c} A_{i}(x)\right)+e A_{0}(x)+\beta m c^{2}\right] \psi=E \psi(x),
$$

where $\psi(x, t)=\mathrm{e}^{\frac{-i E t}{\hbar}}\left(\begin{array}{l}\phi(x) \\ \chi(x)\end{array}\right)$ is the wave function (bi-spinor) in the Dirac representation.

At first we achieve the noncommutativity in space, by the mapping between the noncommutative coordinates $\hat{x}$ and the commutative coordinates $x$ using the ${ }^{*}$-product, with the help of Equation (4) we find

$$
H(\hat{x}, \hat{p}) \star \psi(\hat{x})=\left[c \alpha_{i}\left(\hat{p}_{i}-\frac{e}{c} A_{i}(\hat{x})\right)+e A_{0}(\hat{x})+\beta m c^{2}\right] \star \psi(\hat{x}) .
$$

Consider the electromagnetic potential $(x)=h x$, where $h$ is a constant, the derivations in the Equation (4) roughly turned off in the first order, then Equation (8) can be written as follows

$$
\begin{aligned}
H(\hat{x}, \hat{p}) \star \psi(\hat{x})= & H(x, \hat{p}) \psi(x)+\frac{i}{2} \Theta_{a b} \partial_{a}\left[c \alpha_{i}\left(\hat{p}_{i}-\frac{e}{c} A_{i}(x)\right)\right. \\
& \left.+e A_{0}(\hat{x})+\beta m c^{2}\right] \partial_{b} \psi(x)+\mathrm{O}\left(\Theta^{2}\right) \\
= & E \psi(x),
\end{aligned}
$$

with $\partial_{a}\left(c \alpha_{i} \hat{p}_{i}\right)=\partial_{a}\left(\beta m c^{2}\right)=0$, Equation (9) reduced to

$$
H(x, \hat{p}) \psi(x)-\frac{i e}{2} \theta_{a b} \partial_{a}\left[\hat{\alpha}_{i}\left(A_{i}(x)\right)-A_{0}(\hat{x})\right] \partial_{b} \psi(x)=E \psi(x) .
$$

Anew we achieve the noncommutativity in phase by the mapping between the noncommutative kinetic momentum $\hat{p}$ and the commutative one $p$, using Equation (5) to get the following full noncommutative phase-space Dirac equation

$$
\begin{aligned}
H(\hat{x}, \hat{p}) \star \psi(\hat{x})= & {\left[c \alpha_{i}\left(p_{i}+\frac{1}{2 \hbar} \eta_{i j} x_{j}-\frac{e}{c} A_{i}(x)\right)+e A_{0}(x)+\beta m c^{2}\right.} \\
& \left.-\frac{i e}{2} \Theta_{a b} \partial_{a}\left(\alpha_{i}\left(A_{i}(x)\right)-A_{0}(x) \partial_{b}\right)\right] \psi(x) \\
= & E \psi(x),
\end{aligned}
$$


rewrite the Equation (11) in a more compact form (see Appendix A for the simplification):

$$
\begin{aligned}
\hat{H} \star \psi_{(N C)}= & {\left[c \vec{\alpha}\left(\vec{p}-\frac{e}{c} \vec{A}\right)+e A_{0}+\beta m c^{2}+\frac{c}{\hbar}(\vec{\alpha} \times \vec{x}) \cdot \vec{\eta}\right.} \\
& \left.+\frac{e}{\hbar}\left(\vec{\nabla}\left(\vec{\alpha} \vec{A}-A_{0}\right) \times \vec{p}\right) \cdot \vec{\Theta}\right] \psi_{(N C)} \\
= & E \psi_{(N C)},
\end{aligned}
$$

where $\psi_{(N C)}=\exp \left[\frac{-i\left(E+\frac{c}{\hbar}(\vec{\alpha} \times \vec{x}) \cdot \vec{\eta}\right) t}{\hbar}\right]\left(\begin{array}{l}\phi(\hat{x}) \\ \chi(\hat{x})\end{array}\right)$ is the wave function in

noncommutative phase-space.

\subsection{Foldy-Wouthuysen Transformation in Noncommutative Phase-Space}

Deriving the Schrödinger-Pauli equation in noncommutative phase-space, which is the nonrelativistic limit of the Dirac equation in a simple way using the Foldy-Wouthuysen transformation, this one achieved by a series of successive unitary transformations performed on the phase-space noncommutative Dirac hamiltonian in Equation (12), knowing that it is only applicable to weak fields.

The Dirac hamiltonian in PSNC is given by

$$
\hat{H}=c \vec{\alpha}\left(\vec{p}-\frac{e}{c} \vec{A}\right)+e A_{0}+\beta m c^{2}+\frac{c}{\hbar}(\vec{\alpha} \times \vec{x}) \cdot \vec{\eta}+\frac{e}{\hbar}\left(\vec{\nabla}\left(\vec{\alpha} \vec{A}-A_{0}\right) \times \vec{p}\right) \cdot \vec{\Theta},
$$

in order to perform the FW transformation, we have to rewrite the Dirac hamiltonian Equation (13) to the form:

$$
\hat{H}=\hat{\theta}+\hat{\epsilon}+\beta m c^{2},
$$

where the Dirac hamiltonian is divided into block diagonal and off diagonal parts denoted even operator $\hat{\epsilon}$ and odd operator $\hat{\theta}$ respectively ${ }^{1}$.

$$
\begin{aligned}
& \hat{\epsilon}=e A_{0}-\frac{e}{\hbar}\left(\vec{\nabla}\left(A_{0}\right) \times \vec{p}\right) \cdot \vec{\Theta} \\
& \hat{\theta}=c \vec{\alpha}\left(\vec{p}-\frac{e}{c} \vec{A}\right)+\frac{c}{\hbar}(\vec{\alpha} \times \vec{x}) \cdot \vec{\eta}+\frac{e}{\hbar}(\vec{\nabla}(\vec{\alpha} \vec{A}) \times \vec{p}) \cdot \vec{\Theta},
\end{aligned}
$$

these are defined to satisfy

$$
\beta \hat{\theta}=-\hat{\theta} \beta \text { and } \beta \hat{\epsilon}=\hat{\epsilon} \beta .
$$

Because of the presence of the odd operators (of $\vec{\alpha}$ matrices), the Dirac hamiltonian is not block diagonalized, so that we try to eliminate odd operators from the Dirac hamiltonian, by applying $F W$ transformation

$$
\begin{aligned}
& \psi_{(N C)}^{\prime}=\mathrm{e}^{i \hat{s}} \psi_{(N C)} \\
& \hat{H}^{\prime}=\mathrm{e}^{i \hat{s}} \hat{H} \mathrm{e}^{-i \hat{s}},
\end{aligned}
$$

${ }^{1}$ Odd operators (off diagonal in Pauli-Dirac basis): $\alpha_{i}, \gamma_{i}, \cdots$ even operators (diagonal in Pauli-Dirac basis): $\beta, \Sigma, 1, \cdots$ suffice at the 4 rd order of $\hat{s}$. 
with $\hat{s}$ is Hermitian and it is of the form

$$
\hat{s}=\frac{-i}{2 m c^{2}} \beta \hat{\theta} .
$$

Using the Maclaurin series expansion of $\mathrm{e}^{i \hat{s}}$ defined as $\mathrm{e}^{i \hat{s}}=1+\frac{i \hat{s}}{1 !}+\frac{(i \hat{s})^{2}}{2 !}+\cdots$ in the Equation (17) yields a transformed Dirac hamiltonian (Campbell-Baker-Hausdorff expansion) [23] [24]

$$
\begin{aligned}
\hat{H}^{\prime}= & \hat{H}+i[\hat{s}, \hat{H}]_{-}+\frac{i^{2}}{2 !}\left[\hat{s},[\hat{s}, \hat{H}]_{-}\right]_{-}+\frac{i^{3}}{3 !}\left[\hat{s},\left[\hat{s},[\hat{s}, \hat{H}]_{-}\right]_{-}\right]_{-}+\cdots \\
& +\frac{i^{n}}{n !}\left[\hat{s},\left[\hat{s}, \cdots[\hat{s}, \hat{H}]_{-} \cdots\right]_{-}\right]_{-}+\cdots
\end{aligned}
$$

Writing our hamiltonian, restricting ourselves to terms up to order $1 /\left(m c^{2}\right)^{3}$, thus we suffice at the $4^{\text {rd }}$ order of $\hat{S}$,

$$
\begin{aligned}
\hat{H}^{\prime}= & \hat{H}+i[\hat{s}, \hat{H}]_{-}-\frac{1}{2}\left[\hat{s},[\hat{s}, \hat{H}]_{-}\right]_{-}-\frac{i}{6}\left[\hat{s},\left[\hat{s},[\hat{s}, \hat{H}]_{-}\right]_{-}\right]_{-} \\
& -\frac{1}{24}\left[\hat{s},\left[\hat{s},\left[\hat{s},\left[\hat{s}, \beta m c^{2}\right]_{-}\right]_{-}\right]_{-}\right]_{-}+\cdots
\end{aligned}
$$

Using the properties mentioned in Equation (16) and with Equation (14) and Equation (18), knowing that

$$
\beta[\hat{\theta}, \hat{\epsilon}]_{-}=-[\hat{\theta}, \hat{\epsilon}]_{-} \beta,
$$

we calculate the various commutators of $\hat{s}$ and $\hat{H}$

$$
\begin{gathered}
i[\hat{s}, \hat{H}]_{-}=-\hat{\theta}+\frac{1}{m c^{2}} \hat{\beta} \hat{\theta}^{2}+\frac{\hat{\beta}}{2 m c^{2}}[\hat{\theta}, \hat{\epsilon}]_{-} \\
\frac{i^{2}}{2 !}\left[\hat{s},[\hat{s}, \hat{H}]_{-}\right]_{-}=-\frac{1}{2 m c^{2}} \hat{\beta} \hat{\theta}^{2}-\frac{1}{2 m^{2} c^{4}} \hat{\theta}^{3}+\frac{1}{8 m^{2} c^{4}}\left[\hat{\theta},[\hat{\theta}, \hat{\epsilon}]_{-}\right]_{-} \\
-\frac{i}{6}\left[\hat{s},\left[\hat{s},[\hat{s}, \hat{H}]_{-}\right]_{-}\right]_{-}=\frac{1}{6 m^{2} c^{4}} \hat{\theta}^{3}-\frac{1}{6 m^{3} c^{6}} \hat{\beta} \hat{\theta}^{4}+\frac{1}{48 m^{3} c^{6}} \hat{\beta}\left[\hat{\theta},\left[\hat{\theta},[\hat{\theta}, \hat{\epsilon}]_{-}\right]_{-}\right]_{-}
\end{gathered}
$$

with the same manner we continue, with taking into account only terms of the order $1 /\left(m_{0} c^{2}\right)^{3}$, we get

$$
-\frac{1}{24}\left[\hat{S},\left[\hat{S},\left[\hat{S},\left[\hat{S}, \hat{\beta} m c^{2}\right]_{-}\right]_{-}\right]_{-}\right]_{-} \approx \frac{1}{24 m^{3} c^{6}} \hat{\beta} \hat{\theta}^{4},
$$

by collecting the terms of $\hat{H}^{\prime}$ Equations ((22), (25)), we find

$$
\begin{aligned}
\hat{H}^{\prime}= & \hat{H}-\hat{\theta}+\frac{1}{m c^{2}} \hat{\beta} \hat{\theta}^{2}+\frac{\hat{\beta}}{2 m c^{2}}[\hat{\theta}, \hat{\epsilon}]_{-}-\frac{1}{2 m c^{2}} \hat{\beta} \hat{\theta}^{2}-\frac{1}{2 m^{2} c^{4}} \hat{\theta}^{3} \\
& -\frac{1}{8 m^{2} c^{4}}\left[\hat{\theta},[\hat{\theta}, \hat{\epsilon}]_{-}\right]_{-}+\frac{1}{6 m^{2} c^{4}} \hat{\theta}^{3}-\frac{1}{6 m^{3} c^{6}} \hat{\beta} \hat{\theta}^{4} \\
& -\frac{1}{48 m^{3} c^{6}} \hat{\beta}\left[\hat{\theta},\left[\hat{\theta},[\hat{\theta}, \hat{\epsilon}]_{-}\right]_{-}\right]_{-}+\frac{1}{24 m^{3} c^{6}} \hat{\beta} \hat{\theta}^{4},
\end{aligned}
$$




$$
\begin{aligned}
= & \hat{\epsilon}+\hat{\beta}\left\{m c^{2}+\frac{1}{2 m c^{2}} \hat{\theta}^{2}-\frac{1}{8 m^{3} c^{6}} \hat{\theta}^{4}\right\}+\frac{\hat{\beta}}{2 m c^{2}}[\hat{\theta}, \hat{\epsilon}]_{-}-\frac{1}{3 m^{2} c^{4}} \hat{\theta}^{3} \\
& -\frac{1}{8 m^{2} c^{4}}\left[\hat{\theta},[\hat{\theta}, \hat{\epsilon}]_{-}\right]_{-}-\frac{1}{48 m^{3} c^{6}} \hat{\beta}\left[\hat{\theta},\left[\hat{\theta},[\hat{\theta}, \hat{\epsilon}]_{-}\right]_{-}\right]_{-},
\end{aligned}
$$

as it shown in the Equation (27) the new hamiltonian is not free of the odd operator, the odd part not omit, so By further FW transformation we reduce the odd part of the transformed hamiltonian, so we perform a second transformation, remembering that the product of two even or odd operators is an even operator.

To reduce the odd part of the transformed hamiltonian, thus we chose

$$
\hat{s}^{\prime}=\frac{-i}{2 m c^{2}} \beta \hat{\theta}^{\prime}
$$

in where

$$
\hat{H}^{\prime}=\hat{H}+\hat{\theta}^{\prime}+\hat{\epsilon}^{\prime}+\hat{\beta} m c^{2}
$$

with

$$
\begin{gathered}
\hat{\theta}^{\prime}=\frac{\hat{\beta}}{2 m c^{2}}[\hat{\theta}, \hat{\epsilon}]_{-}-\frac{1}{3 m^{2} c^{4}} \hat{\theta}^{3}-\frac{1}{48 m^{3} c^{6}} \hat{\beta}\left[\hat{\theta},\left[\hat{\theta},[\hat{\theta}, \hat{\epsilon}]_{-}\right]_{-}\right]_{-}, \\
\hat{\epsilon}^{\prime}=\hat{\epsilon}+\frac{1}{m c^{2}} \hat{\beta} \hat{\theta}^{2}-\frac{1}{8 m^{3} c^{6}} \hat{\beta} \hat{\theta}^{4}-\frac{1}{8 m^{2} c^{4}}\left[\hat{\theta},[\hat{\theta}, \hat{\epsilon}]_{-}\right]_{-}, \\
\hat{H}^{\prime \prime}=\mathrm{e}^{i \hat{s}^{\prime}} \hat{H}^{\prime} \mathrm{e}^{-i \hat{s}^{\prime}}, \\
\hat{H}^{\prime}+i\left[\hat{s}^{\prime}, \hat{H}^{\prime}\right]_{-}-\frac{1}{2}\left[\hat{s}^{\prime},\left[\hat{s}^{\prime}, \hat{H}^{\prime}\right]_{-}\right]_{-}-\frac{i}{6}\left[\hat{s}^{\prime},\left[\hat{s}^{\prime},\left[\hat{s}^{\prime}, \hat{H}^{\prime}\right]_{-}\right]_{-}\right]_{-} \\
-\frac{1}{24}\left[\hat{s}^{\prime},\left[\hat{s}^{\prime},\left[\hat{s}^{\prime},\left[\hat{s}^{\prime}, \beta m c^{2}\right]_{-}\right]_{-}\right]_{-}\right]_{-}+\cdots
\end{gathered}
$$

We restrict ourselves to terms up to order $1 /\left(m c^{2}\right)^{3}$, as in the first FW transformation (knowing that $\hat{\theta}^{\prime} \sim \frac{1}{m c^{2}}$ ), so that the new hamiltonian is

$$
\hat{H}^{\prime \prime}=\hat{\beta} m c^{2}+\hat{\epsilon}^{\prime}+\frac{\hat{\beta}}{2 m c^{2}}\left[\hat{\theta}^{\prime}, \hat{\epsilon}^{\prime}\right]_{-}+\frac{1}{2 m c^{2}} \hat{\beta} \hat{\theta}^{\prime 2}-\frac{1}{2 m^{2} c^{4}} \hat{\theta}^{\prime 3}-\frac{1}{8 m^{2} c^{4}}\left[\hat{\theta}^{\prime},\left[\hat{\theta}^{\prime}, \hat{\epsilon}^{\prime}\right]_{-}\right]_{-} .
$$

The terms proportional to $\hat{\theta}^{\prime 3}$ and $\hat{\theta}^{\prime 2}$ contain large powers of $1 / m c^{2}$, therefore they can be neglected, more precisely we take into account only terms of order that we restrict ourselves in the expansion, and thus Equation (34) is given by

$$
\hat{H}^{\prime \prime} \simeq \hat{\beta} m c^{2}+\hat{\epsilon}^{\prime}+\frac{\hat{\beta}}{2 m c^{2}}\left[\hat{\theta}^{\prime}, \hat{\epsilon}^{\prime}\right]_{-}=\hat{\beta} m c^{2}+\hat{\epsilon}^{\prime}+\hat{\theta}^{\prime \prime},
$$

$\hat{H}^{\prime \prime}$ is not yet free of the odd operators, we apply a third FW transformation to eliminate $\hat{\theta}^{\prime \prime}$,

$$
\hat{H}^{\prime \prime \prime}=\mathrm{e}^{i \hat{s}^{\prime \prime}} \hat{H}^{\prime \prime} \mathrm{e}^{-i \hat{s}^{\prime \prime}}
$$

with

$$
\hat{s}^{\prime \prime}=\frac{-i}{2 m c^{2}} \beta \hat{\theta}^{\prime \prime}
$$


so that

$$
\hat{H}^{\prime \prime \prime} \simeq \hat{\beta} m c^{2}+\hat{\epsilon}^{\prime}=\hat{\beta} m c^{2}+\hat{\epsilon}+\frac{1}{m c^{2}} \hat{\beta} \hat{\theta}^{2}-\frac{1}{8 m^{3} c^{6}} \hat{\beta} \hat{\theta}^{4}-\frac{1}{8 m^{2} c^{4}}\left[\hat{\theta},[\hat{\theta}, \hat{\epsilon}]_{-}\right]_{-} .
$$

Finally the transformed hamiltonian is completely free of odd operators, next we calculate the various terms of the Equation (38), according to Equation (15), we make use of the following known relations for three arbitrary vectors $\vec{A}, \vec{B}$ and $\vec{C}$ :

$$
\vec{A} \cdot(\vec{B} \times \vec{C})=\vec{B} \cdot(\vec{C} \times \vec{A})=\vec{C} \cdot(\vec{A} \times \vec{B}),(\vec{A} \times \vec{B}) \cdot \vec{C}=(\vec{B} \times \vec{C}) \cdot \vec{A}=(\vec{C} \times \vec{A}) \cdot \vec{B},
$$

the Equation (15) becomes

$$
\hat{\theta}=c \vec{\alpha}\left(\vec{p}-\frac{e}{c} \vec{A}\right)+\frac{c}{\hbar} \vec{\alpha}\left((\vec{x} \times \vec{\eta})+\frac{e}{c} \vec{\nabla} \vec{A}(\vec{p} \times \vec{\Theta})\right)=c \vec{\alpha}\left(\vec{p}-\frac{e}{c} \vec{A}\right)+\frac{c}{\hbar} \vec{\alpha} \vec{\Omega},
$$

with

$$
\vec{\Omega}=\vec{x} \times \vec{\eta}+\frac{e}{c} \vec{\nabla} \vec{A}(\vec{p} \times \vec{\Theta}),
$$

yields

$$
\hat{\theta}^{2}=c^{2}\left(\vec{\alpha}\left(\vec{p}-\frac{e}{c} \vec{A}\right)\right)^{2}+\frac{2 c}{\hbar} \vec{\alpha}\left(\vec{p}-\frac{e}{c} \vec{A}\right) \vec{\alpha} \vec{\Omega}+\frac{c^{2}}{\hbar^{2}}(\vec{\alpha} \vec{\Omega})^{2},
$$

using the following relation ${ }^{2}$

$$
(\vec{\alpha} \vec{A})(\vec{\alpha} \vec{B})=\vec{A} \vec{B}+i \vec{\Sigma} \cdot(\vec{A} \times \vec{B}),
$$

we obtain

$$
\begin{aligned}
\hat{\theta}^{2}= & c^{2}\left(\vec{\alpha}\left(\vec{p}-\frac{e}{c} \vec{A}\right)\right)^{2}+\frac{2 c}{\hbar}\left(\vec{p}-\frac{e}{c} \vec{A}\right) \vec{\Omega}+\frac{c^{2}}{\hbar^{2}} \vec{\Omega}^{2} \\
& +i c^{2} \vec{\Sigma} \cdot\left[\frac{i \hbar e}{c} \vec{B}+\frac{2}{\hbar}\left(\vec{p}-\frac{e}{c} \vec{A}\right) \times \vec{\Omega}+\frac{1}{\hbar} \vec{\Omega} \times \vec{\Omega}\right]
\end{aligned}
$$

and

$$
\begin{aligned}
& \frac{1}{8 m^{2} c^{4}}[\hat{\theta}, \hat{\epsilon}]_{-}=-\frac{i \hbar}{8 m^{2} c^{4}} c \vec{\alpha} \vec{\nabla} A_{0} \\
& +\frac{1}{8 m^{2} c^{4}}\left\{\left[\frac{e}{\hbar}\left(\vec{\nabla}\left(A_{0}\right) \times \vec{p}\right) \cdot \vec{\Theta}, c \vec{\alpha}\left(\vec{p}-\frac{e}{c} \vec{A}\right)+\frac{c}{\hbar} \vec{\alpha} \vec{\Omega}\right]_{-}+\left[\frac{c}{\hbar} \vec{\alpha} \vec{\Omega}, e A_{0}\right]_{-}\right\},
\end{aligned}
$$

for $A_{0}=V$ we have $\vec{\nabla} V=-\vec{E}$ and using Equation (45) we have

$$
\begin{aligned}
& {\left[\hat{\theta},[\hat{\theta}, \hat{\epsilon}]_{-}\right]_{-}=\frac{e \hbar^{2} c^{2}}{8 m^{2} c^{4}} \vec{\nabla} \vec{E}+\frac{i e \hbar^{2} c^{2}}{8 m^{2} c^{4}} \vec{\Sigma} \cdot \vec{\nabla} \times \vec{E}} \\
& +\frac{e \hbar}{4 m^{2} c^{4}} \vec{\Sigma} \cdot \vec{E} \times \vec{p}+\left[\frac{c}{\hbar} \vec{\alpha} \vec{\Omega}, \frac{i \hbar}{8 m^{2} c^{4}} c \vec{\alpha} \vec{E}\right]_{-} \\
& +\left[\hat{\theta}, \frac{1}{8 m^{2} c^{4}}\left\{\left[c \vec{\alpha}\left(\vec{p}-\frac{e}{c} \vec{A}\right)-\frac{c}{\hbar} \vec{\alpha} \vec{\Omega}, \frac{e}{\hbar}\left(\vec{\nabla}\left(A_{0}\right) \times \vec{p}\right) \cdot \vec{\Theta}\right]_{-}+\left[\frac{c}{\hbar} \vec{\alpha} \vec{\Omega}, e A_{0}\right]\right\}\right], \\
& { }^{2} \alpha^{i} \alpha^{j}=\alpha^{i} \beta^{2} \alpha^{j}=-\beta \alpha^{i} \beta \alpha^{j}=\gamma^{i} \gamma^{j}=-\frac{1}{2}\left(\left\{\gamma^{i}, \gamma^{j}\right\}+\left[\gamma^{i}, \gamma^{j}\right]\right)=-g^{i j}+i \epsilon^{i j k} \Sigma^{k}=\delta^{i j}+i \epsilon^{i j k} \Sigma^{k}
\end{aligned}
$$


using Equation (43) we simplify Equation (46) (see Appendix B), to have

$$
\begin{aligned}
& {\left[\hat{\theta},[\hat{\theta}, \hat{\epsilon}]_{-}\right]_{-}=\frac{e \hbar^{2} c^{2}}{8 m^{2} c^{4}} \vec{\nabla} \vec{E}+\frac{i e \hbar^{2} c^{2}}{8 m^{2} c^{4}} \vec{\Sigma} \cdot \vec{\nabla} \times \vec{E}+\frac{e \hbar}{4 m^{2} c^{4}} \vec{\Sigma} \cdot \vec{E} \times \vec{p}} \\
& +\frac{i c^{2}}{8 m^{2} c^{4}}\left([\vec{\Omega}, \vec{E}]_{-}+2 i \vec{\Sigma}(\vec{\Omega} \times \vec{E})\right)+\frac{1}{8 m^{2} c^{4}} \frac{e}{\hbar}\left[\left(c\left(\vec{p}-\frac{e}{c} \vec{A}\right)+\frac{c}{\hbar} \vec{\Omega}\right),\right. \\
& \left.\left[\left(c\left(\vec{p}-\frac{e}{c} \vec{A}\right)-\frac{c}{\hbar} \vec{\Omega}\right),(\vec{E} \times \vec{p}) \cdot \vec{\Theta}\right]_{-}-c[\vec{\Omega}, V]_{-}\right]_{-} \\
& +\frac{1}{8 m^{2} c^{4}} \frac{e}{\hbar} i \vec{\Sigma}\left\{-2(\vec{E} \times \vec{p}) \cdot \vec{\Theta} \cdot\left(c\left(\vec{p}-\frac{e}{c} \vec{A}\right)-\frac{c}{\hbar} \vec{\Omega}\right)\right. \\
& \times\left(c\left(\vec{p}-\frac{e}{c} \vec{A}\right)+\frac{c}{\hbar} \vec{\Omega}\right)+2 c[\vec{\Omega}, V] \times\left(c\left(\vec{p}-\frac{e}{c} \vec{A}\right)+\frac{c}{\hbar} \vec{\Omega}\right) \\
& \left.+\left[\left(c\left(\vec{p}-\frac{e}{c} \vec{A}\right)+\frac{c}{\hbar} \vec{\Omega}\right) \times\left(c\left(\vec{p}-\frac{e}{c} \vec{A}\right)-\frac{c}{\hbar} \vec{\Omega}\right),(\vec{E} \times \vec{p}) \cdot \vec{\Theta}\right]\right\} .
\end{aligned}
$$

Adding the various contributions Equations ((44), (47)), in Equation (38), with assuming $\frac{1}{8 m^{3} c^{6}} \hat{\theta}^{4} \sim \frac{1}{8 m^{3} c^{6}} \vec{p}^{4}$, and For $\psi_{F W}=\left(\begin{array}{c}\varphi \\ 0\end{array}\right)$ the Schrödinger-Pauli equation is

$$
\begin{aligned}
& i \hbar \frac{\partial}{\partial t} \varphi=\left\{\hat { \beta } \left(m c^{2}+\frac{1}{2 m}\left(\vec{p}-\frac{e}{c} \vec{A}\right)^{2}-\frac{1}{8 m^{3} c^{6}} \vec{p}^{4}+\frac{1}{m \hbar}\left(\vec{p}-\frac{e}{c} \vec{A}\right) \vec{\Omega}+\frac{1}{2 m \hbar^{2}} \vec{\Omega}^{2}\right.\right. \\
& \left.+\frac{i}{2 m} \vec{\Sigma}\left(\frac{2}{\hbar}\left(\vec{p}-\frac{e}{c} \vec{A}\right) \times \vec{\Omega}+\frac{1}{\hbar^{2}} \vec{\Omega} \times \vec{\Omega}\right)\right)+e V-\frac{e \hbar}{2 m c} \hat{\beta} \vec{\Sigma} \vec{B}-\frac{i e \hbar^{2} c^{2}}{8 m^{2} c^{4}} \vec{\Sigma} \cdot \vec{\nabla} \times \vec{E} \\
& -\frac{e \hbar}{4 m^{2} c^{4}} \vec{\Sigma} \cdot \vec{E} \times \vec{p}+\frac{e}{\hbar}(\vec{E} \times \vec{p}) \cdot \vec{\Theta}-\frac{e \hbar^{2} c^{2}}{8 m^{2} c^{4}} \vec{\nabla} \vec{E}-\frac{i c^{2}}{8 m^{2} c^{4}}([\vec{\Omega}, \vec{E}]+2 i \vec{\Sigma}(\vec{\Omega} \times \vec{E})) \\
& -\frac{1}{8 m^{2} c^{4}} \frac{e}{\hbar}\left\{\left[\left(c\left(\vec{p}-\frac{e}{c} \vec{A}\right)+\frac{c}{\hbar} \vec{\Omega}\right) \times\left(c\left(\vec{p}-\frac{e}{c} \vec{A}\right)-\frac{c}{\hbar} \vec{\Omega}\right),(\vec{E} \times \vec{p}) \cdot \vec{\Theta}\right]-\right. \\
& +2 c[\vec{\Omega}, V] \times\left(c\left(\vec{p}-\frac{e}{c} \vec{A}\right)+\frac{c}{\hbar} \vec{\Omega}\right)-2(\vec{E} \times \vec{p}) \cdot \vec{\Theta}\left(c\left(\vec{p}-\frac{e}{c} \vec{A}\right)-\frac{c}{\hbar} \vec{\Omega}\right) \\
& \left.\left.\times\left(c\left(\vec{p}-\frac{e}{c} \vec{A}\right)+\frac{c}{\hbar} \vec{\Omega}\right)\right\}\right\} .
\end{aligned}
$$

The reason of the explicit noncommutative terms entangled in the obtained Schrödinger-Pauli Equation (48), (in FW representation) is that the effect of the noncommutativity in the Dirac equation appears as a kind of potential which depends on noncommutativity parameters $(\eta$, and $\Theta$ ), then after applying the nonrelativistic limit that potential is the responsible on generating a new terms and a modified known terms, which contain the noncommutative parameters (reduced in $\vec{\Omega}$ ), in Equation (48), where terms in the first parenthesis describe the NC nonrelativistic kinetic energy, and its first NC nonrelativistic correction (at least to the order of approximation we have considered), this is manifested as a terms contain phase-space NC parameters added to the known terms, then 
successive two terms describe the electrostatic energy and the magnetic dipole energy, thereafter $-\frac{e \hbar}{4 m^{2} c^{4}} \vec{\Sigma} \cdot \vec{E} \times \vec{p}+\frac{e}{\hbar}(\vec{E} \times \vec{p}) \cdot \vec{\Theta}$, represents the $\Theta$-modified spin-orbit interaction term, in a spherically symmetric potential, with

$$
\vec{\nabla} \times \vec{E}=0, \vec{E}=-\vec{\nabla} V(r)=-\frac{1}{r} \frac{\partial V}{\partial r} \vec{r} \text {, and } \vec{r} \times \vec{p}=\vec{L},
$$$$
\Theta \text {-modified spin-orbit term }=\frac{e \hbar}{4 m^{2} c^{4}} \frac{1}{r} \frac{\partial V}{\partial r} \vec{\Sigma} \cdot \vec{L}-\frac{e}{\hbar} \frac{1}{r} \frac{\partial V}{\partial r} \vec{L} \cdot \vec{\Theta} \text {. }
$$

Next term in Equation (48), $-\frac{e \hbar^{2} c^{2}}{8 m^{2} c^{4}} \vec{\nabla} \vec{E}-\frac{i c^{2}}{8 m^{2} c^{4}}\left([\vec{\Omega}, \vec{E}]_{-}+2 i \vec{\Sigma}(\vec{\Omega} \times \vec{E})\right)$, represents the $\Theta \eta$-modified Darwin term (attributed to the Zitterbewegung [25] [26] [27]), with $\vec{E}=-\vec{\nabla} V(r)$ and $\vec{A}=0, \vec{\Omega}=\vec{x} \times \vec{\eta}$,

$\Theta \eta$-modified Darwin term

$$
=-\frac{e \hbar^{2} c^{2}}{8 m^{2} c^{4}} \vec{\nabla} V-\frac{i c^{2}}{8 m^{2} c^{4}}\left([\vec{x} \times \vec{\eta}, \vec{\nabla} V]_{-}+2 i \vec{\Sigma}(\vec{x} \times \vec{\eta} \times \vec{\nabla} V)\right) .
$$

The other terms in the Equation (48) represent the NC Schrödinger-Pauli equation corrections.

Essentially the intriguing part of our result is the fact that noncommutative effects grant a $\Theta \eta$-modified terms entangled in the obtained NC Schrödinger-Pauli equation.

Under the condition that space-space and momentum-momentum are all commutative (namely, $\eta=0, \Theta=0$ ) the results return to that of usual quantum mechanics.

\section{Conclusions}

In conclusion, the phase-space noncommutativity effect is introduced in the Dirac equation and subsequently the Foldy-Wouthuysen transformation is exploited to reduce the system in presence of electromagnetic field to a nonrelativistic regime, which gives the Schrödinger-Pauli equation.

Knowing that the phase-space noncommutativity effect is introduced by applying both of the Bopp-shift linear translation method, and the Moyal-Weyl product.

The usage of the FW representation in most cases allows one to reduce the problem of finding a classical limit of relativistic quantum mechanical equations to the replacement of operators in the hamiltonian of the quantum mechanical equations of motion by the respective classical quantities, even with noncommutativity in phase and space, and the effects of the latter are manifested in the various terms of the obtained hamiltonian.

\section{Conflicts of Interest}

The authors declare no conflicts of interest regarding the publication of this paper. 


\section{References}

[1] Foldy, L.L. and Wouthuysen, S.A. (1950) Physical Review, 78, 29. https://doi.org/10.1103/PhysRev.78.29

[2] Greiner, W. (2000) Quantum Mechanics an Introduction, 4th Edition, Vol. I, Springer, Berlin.

[3] Greiner, W. (1994) Quantum Mechanics. 3rd Edition, Springer, Berlin, Heidelberg.

[4] Davydov, A.S. (1965) Quantum Mechanics. 2nd Edition, Pergamon, Oxford.

[5] Messiah, A. (1968) Quantum Mechanics, Vol. II. Wiley, New York.

[6] Costello, J.P. and McKellar, B.H.J. (1995) American Journal of Physics, 63, 1119-1121. https://doi.org/10.1119/1.18017

[7] Silenko, A.J. (2003) Journal of Mathematical Physics, 44, 2952. https://doi.org/10.1063/1.1579991

[8] Silenko, A.J. (2008) Physical Review A, 77, Article ID: 012116. https://doi.org/10.1103/PhysRevA.77.012116

[9] Nikitin, A.G. (1998) Journal of Physics A: Mathematical and General, 31, 3297-3300. https://doi.org/10.1088/0305-4470/31/14/015

[10] Delduc, F., Duret, Q., Gieres, F. and Lefrancois, M. (2008) Journal of Physics Conference Series, 103, Article ID: 012020. https://doi.org/10.1088/1742-6596/103/1/012020

[11] Douglas, M.R. and Nekrasov, N.A. (2001) Reviews of Modern Physics, 73, 977-1029. https://doi.org/10.1103/RevModPhys.73.977

[12] Connes, A., Douglas, M.R. and Schwarz, A. (1998) JHEP, 9802, 003. https://doi.org/10.1088/1126-6708/1998/02/003

[13] Seiberg, N. and Witten, E. (1999) JHEP, 9909, 032. https://doi.org/10.1088/1126-6708/1999/09/032

[14] Chaichian, M., Demichev, A. and Presnajder, P. (2000) Nuclear Physics B, 567, 360. https://doi.org/10.1016/S0550-3213(99)00664-1

[15] Mirza, B. and Mohadesi, M. (2014) Communications in Theoretical Physics, 42, 664-668. http://iopscience.iop.org/0253-6102/42/5/664

[16] Bastos, C., Bertolami, O., Dias, N.C. and Prata, J.N. (2008) Journal of Mathematical Physics, 49, Article ID: 072101. https://doi.org/10.1063/1.2944996

[17] Eftekharzadeh, A. and Hu, B.L. (2005) Brazilian Journal of Physics, 35, 333-342. https://doi.org/10.1590/S0103-97332005000200019

[18] Li, K., Wang, J. and Chen, C. (2005) Modern Physics Letters A, 20, 2165-2174. https://doi.org/10.1142/S0217732305017421

[19] Jiang, X., Long, C. and Qin, S. (2013) Journal of Modern Physics, 4, 940-944. https://www.doi.org/10.4236/jmp.2013.47126

[20] Hassanabadi, H., Molaee, Z. and Zarrinkamar, S. (2014) Advances in High Energy Physics, 2014, Article ID: 459345. http://dx.doi.org/10.1155/2014/459345

[21] Adorno, T.C., Baldiotti, M.C., Chaichian, M., Gitman, D.M. and Tureanu, A. (2009) Physics Letters B, 682, 235-239. https://doi.org/10.1016/j.physletb.2009.11.003

[22] Bertolami, O. and Queiroz, R. (2011) Physics Letters A, 375, 4116-4119. https://doi.org/10.1016/j.physleta.2011.09.053

[23] Schwabl, F. (1995) Quantum Mechanics. Springer, Berlin.

[24] Kurlin, V. (2007) Journal of Lie Theory, 17, 525-538. arXiv:math/0606330. 
[25] Hestenes, D. (1990) Foundations of Physics, 20, 1213-1232. https://doi.org/10.1007/BF01889466

[26] Huang, K. (1952) American Journal of Physics, 20, 479. https://doi.org/10.1119/1.1933296

[27] Barut, A.O. and Bracken, A.J. (1981) Physical Review D, 23, 2454. https://doi.org/10.1103/PhysRevD.23.2454 


\section{Appendix A: Moving from Equation (11) to Equation (12)}

The simplification to move from Equation (11) to Equation (12):

Using $\eta_{i j}=\eta \epsilon_{i j}$ and $\eta_{k}=\frac{1}{2} \epsilon_{k i j} \eta_{i j}$, we find:

$$
\begin{aligned}
\alpha_{i} \frac{c}{2 \hbar} \eta_{i j} X_{j} & =\frac{c}{2 \hbar}\left(2 \eta_{k} \epsilon_{k i j}^{-1}\right) \alpha_{i} X_{j} \\
& =\frac{c}{\hbar} \eta_{k} \epsilon_{k i j} \alpha_{i} X_{j},
\end{aligned}
$$

where $\epsilon_{k i j}=\epsilon_{i j k}$, knowing that $(U \times V)_{\mu}=\epsilon_{\mu \nu \lambda} U_{\nu} V_{\lambda}$,

$$
\begin{aligned}
\frac{c}{\hbar} \eta_{k} \epsilon_{k i j} \alpha_{i} X_{j} & =\frac{c}{\hbar}(\vec{\alpha} \times \vec{X})_{k} \eta_{k} \\
& =\frac{c}{\hbar}(\vec{\alpha} \times \vec{X}) \vec{\eta},
\end{aligned}
$$

with the same manner we prove that

$$
\begin{aligned}
& -i e \Theta_{k} \epsilon_{a b k} \partial_{a}\left(\vec{\alpha} \vec{A}-A_{0}\right) \partial_{b} \\
& =-i e \frac{\hbar}{\hbar} \Theta_{k} \epsilon_{a b k} \partial_{a}\left(\vec{\alpha} \vec{A}-A_{0}\right) \partial_{b} \\
& =\frac{e}{\hbar}\left(\vec{\nabla} \cdot\left(\vec{\alpha} \vec{A}-A_{0}\right) \times \vec{p}\right) \cdot \vec{\Theta} .
\end{aligned}
$$

Appendix B: The Elimination of $\vec{\alpha}$ from the Two Last Terms in Equation (46)

Using The Equation (43) we find

$$
\begin{aligned}
{\left[\frac{c}{\hbar} \vec{\alpha} \vec{\Omega}, \frac{i \hbar}{8 m^{2} c^{4}} c \vec{\alpha} \vec{E}\right]_{-} } & =\frac{c}{\hbar} \vec{\alpha} \vec{\Omega} \frac{i \hbar}{8 m^{2} c^{4}} c \vec{\alpha} \vec{E}-\frac{i \hbar}{8 m^{2} c^{4}} c \vec{\alpha} \vec{E} \frac{c}{\hbar} \vec{\alpha} \vec{\Omega} \\
& =\frac{c^{2}}{\hbar} \frac{i \hbar}{8 m^{2} c^{4}}\{\vec{\alpha} \vec{\Omega} \vec{\alpha} \vec{E}-\vec{\alpha} \vec{E} \vec{\alpha} \vec{\Omega}\} \\
& =\frac{i c^{2}}{8 m^{2} c^{4}}\{\vec{\Omega} \vec{E}+i \vec{\Sigma}(\vec{\Omega} \times \vec{E})-\vec{E} \vec{\Omega}-i \vec{\Sigma}(\vec{E} \times \vec{\Omega})\} \\
& =\frac{i c^{2}}{8 m^{2} c^{4}}\left\{[\vec{\Omega}, \vec{E}]_{-}+i 2 \vec{\Sigma}(\vec{\Omega} \times \vec{E})\right\} .
\end{aligned}
$$

And for the second term

$$
\begin{aligned}
& {\left[c \vec{\alpha}\left(\vec{p}-\frac{e}{c} \vec{A}\right)+\frac{c}{\hbar} \vec{\alpha} \vec{\Omega}, \frac{-1}{8 m^{2} c^{4}}\left[\left(c \vec{\alpha}\left(\vec{p}-\frac{e}{c} \vec{A}\right)-\frac{c}{\hbar} \vec{\alpha} \vec{\Omega}\right), \frac{e}{\hbar}(-\vec{E} \times \vec{p}) \cdot \vec{\Theta}\right]_{-}\right.} \\
& \left.-\frac{1}{8 m^{2} c^{4}}\left[\frac{c}{\hbar} \vec{\alpha} \vec{\Omega}, e V\right]_{-}\right]_{-} \\
& =\left[c \vec{\alpha}\left(\vec{p}-\frac{e}{c} \vec{A}\right)+\frac{c}{\hbar} \vec{\alpha} \vec{\Omega}, \frac{-1}{8 m^{2} c^{4}} \frac{c}{\hbar} e \vec{\alpha}[\vec{\Omega}, V]_{-}\right]_{-} \\
& +\left[c \vec{\alpha}\left(\vec{p}-\frac{e}{c} \vec{A}\right)+\frac{c}{\hbar} \vec{\alpha} \vec{\Omega}, \frac{-1}{8 m^{2} c^{4}}\left[\left(c \vec{\alpha}\left(\vec{p}-\frac{e}{c} \vec{A}\right)-\frac{c}{\hbar} \vec{\alpha} \vec{\Omega}\right), \frac{e}{\hbar}\left(\vec{\nabla}\left(A_{0}\right) \times \vec{p}\right) \cdot \vec{\Theta}\right]\right]_{-},
\end{aligned}
$$

we start with the $1^{\text {st }}$ term of the above Equation (55) 


$$
\begin{aligned}
& {\left[c \vec{\alpha}\left(\vec{p}-\frac{e}{c} \vec{A}\right)+\frac{c}{\hbar} \vec{\alpha} \vec{\Omega}, \frac{-1}{8 m^{2} c^{4}} \frac{c}{\hbar} e \vec{\alpha}[\vec{\Omega}, V]_{-}\right]_{-}} \\
& =-\vec{\alpha}\left(c\left(\vec{p}-\frac{e}{c} \vec{A}\right)+\frac{c}{\hbar} \vec{\Omega}\right)\left(\frac{1}{8 m^{2} c^{4}} \frac{c}{\hbar} e \vec{\alpha}[\vec{\Omega}, V]_{-}\right) \\
& \quad+\left(\frac{1}{8 m^{2} c^{4}} \frac{c}{\hbar} e \vec{\alpha}[\vec{\Omega}, V]_{-}\right) \vec{\alpha}\left(c\left(\vec{p}-\frac{e}{c} \vec{A}\right)+\frac{c}{\hbar} \vec{\Omega}\right) \\
& =\left[\frac{1}{8 m^{2} c^{4}} \frac{c}{\hbar} e \vec{\alpha}[\vec{\Omega}, V],\left(c\left(\vec{p}-\frac{e}{c} \vec{A}\right)+\frac{c}{\hbar} \vec{\Omega}\right)\right]_{-} \\
& \quad+2 i \frac{1}{8 m^{2} c^{4}} \frac{c}{\hbar} e \vec{\Sigma}\left([\vec{\Omega}, V]_{-}\right) \times\left(c\left(\vec{p}-\frac{e}{c} \vec{A}\right)+\frac{c}{\hbar} \vec{\Omega}\right),
\end{aligned}
$$

using Equation (39), we continue with the $2^{\text {nd }}$ term,

$$
\begin{aligned}
& {\left[c \vec{\alpha}\left(\vec{p}-\frac{e}{c} \vec{A}\right)+\frac{c}{\hbar} \vec{\alpha} \vec{\Omega}, \frac{1}{8 m^{2} c^{4}}\left[\left(c \vec{\alpha}\left(\vec{p}-\frac{e}{c} \vec{A}\right)-\frac{c}{\hbar} \vec{\alpha} \vec{\Omega}\right), \frac{e}{\hbar}(\vec{E} \times \vec{p}) \cdot \vec{\Theta}\right]\right]} \\
& =\frac{1}{8 m^{2} c^{4}} \frac{e}{\hbar}\left\{( c ( \vec { p } - \frac { e } { c } \vec { A } ) + \frac { c } { \hbar } \vec { \Omega } ) \left\{\left(c\left(\vec{p}-\frac{e}{c} \vec{A}\right)-\frac{c}{\hbar} \vec{\Omega}\right)(\vec{E} \times \vec{p}) \cdot \vec{\Theta}\right.\right. \\
& \left.-(\vec{E} \times \vec{p}) \cdot \vec{\Theta}\left(c\left(\vec{p}-\frac{e}{c} \vec{A}\right)-\frac{c}{\hbar} \vec{\Omega}\right)\right\}-\left\{\left(c\left(\vec{p}-\frac{e}{c} \vec{A}\right)-\frac{c}{\hbar} \vec{\Omega}\right)(\vec{E} \times \vec{p}) \cdot \vec{\Theta}\right. \\
& \left.\left.-(\vec{E} \times \vec{p}) \cdot \vec{\Theta}\left(c\left(\vec{p}-\frac{e}{c} \vec{A}\right)-\frac{c}{\hbar} \vec{\Omega}\right)\right\}\left(c\left(\vec{p}-\frac{e}{c} \vec{A}\right)+\frac{c}{\hbar} \vec{\Omega}\right)\right\} \\
& +\frac{1}{8 m^{2} c^{4}} \frac{e}{\hbar} i \vec{\Sigma}\left\{\left(c\left(\vec{p}-\frac{e}{c} \vec{A}\right)+\frac{c}{\hbar} \vec{\Omega}\right)\left(c\left(\vec{p}-\frac{e}{c} \vec{A}\right)-\frac{c}{\hbar} \vec{\Omega}\right)(\vec{E} \times \vec{p}) \cdot \vec{\Theta}\right. \\
& -\left(c\left(\vec{p}-\frac{e}{c} \vec{A}\right)+\frac{c}{\hbar} \vec{\Omega}\right)(\vec{E} \times \vec{p}) \cdot \vec{\Theta}\left(c\left(\vec{p}-\frac{e}{c} \vec{A}\right)-\frac{c}{\hbar} \vec{\Omega}\right) \\
& -(\vec{E} \times \vec{p}) \cdot \vec{\Theta}\left(c\left(\vec{p}-\frac{e}{c} \vec{A}\right)-\frac{c}{\hbar} \vec{\Omega}\right)\left(c\left(\vec{p}-\frac{e}{c} \vec{A}\right)+\frac{c}{\hbar} \vec{\Omega}\right) \\
& \left.-(\vec{E} \times \vec{p}) \cdot \vec{\Theta}\left(c\left(\vec{p}-\frac{e}{c} \vec{A}\right)-\frac{c}{\hbar} \vec{\Omega}\right) \times\left(c\left(\vec{p}-\frac{e}{c} \vec{A}\right)+\frac{c}{\hbar} \vec{\Omega}\right)\right\} \\
& =\frac{1}{8 m^{2} c^{4}} \frac{e}{\hbar}\left\{\left(c\left(\vec{p}-\frac{e}{c} \vec{A}\right)+\frac{c}{\hbar} \vec{\Omega}\right)\left\{\left[\left(c\left(\vec{p}-\frac{e}{c} \vec{A}\right)-\frac{c}{\hbar} \vec{\Omega}\right),(\vec{E} \times \vec{p}) \cdot \vec{\Theta}\right]\right\}\right. \\
& \left.-\left\{\left[\left(c\left(\vec{p}-\frac{e}{c} \vec{A}\right)-\frac{c}{\hbar} \vec{\Omega}\right),(\vec{E} \times \vec{p}) \cdot \vec{\Theta}\right]_{-}\right\}\left(c\left(\vec{p}-\frac{e}{c} \vec{A}\right)+\frac{c}{\hbar} \vec{\Omega}\right)\right\} \\
& +\frac{1}{8 m^{2} c^{4}} \frac{e}{\hbar} i \vec{\Sigma}\left\{\left(c\left(\vec{p}-\frac{e}{c} \vec{A}\right)+\frac{c}{\hbar} \vec{\Omega}\right) \times\left(c\left(\vec{p}-\frac{e}{c} \vec{A}\right)-\frac{c}{\hbar} \vec{\Omega}\right)(\vec{E} \times \vec{p}) \cdot \vec{\Theta}\right. \\
& -(\vec{E} \times \vec{p}) \cdot \vec{\Theta}\left(c\left(\vec{p}-\frac{e}{c} \vec{A}\right)-\frac{c}{\hbar} \vec{\Omega}\right) \times\left(c\left(\vec{p}-\frac{e}{c} \vec{A}\right)+\frac{c}{\hbar} \vec{\Omega}\right) \\
& -(\vec{E} \times \vec{p}) \cdot \vec{\Theta}\left(c\left(\vec{p}-\frac{e}{c} \vec{A}\right)+\frac{c}{\hbar} \vec{\Omega}\right) \times\left(c\left(\vec{p}-\frac{e}{c} \vec{A}\right)-\frac{c}{\hbar} \vec{\Omega}\right) \\
& \left.-(\vec{E} \times \vec{p}) \cdot \vec{\Theta}\left(c\left(\vec{p}-\frac{e}{c} \vec{A}\right)-\frac{c}{\hbar} \vec{\Omega}\right) \times\left(c\left(\vec{p}-\frac{e}{c} \vec{A}\right)+\frac{c}{\hbar} \vec{\Omega}\right)\right\},
\end{aligned}
$$


finally we find

$$
\begin{aligned}
& \frac{1}{8 m^{2} c^{4}} \frac{e}{\hbar}\left[\left(c\left(\vec{p}-\frac{e}{c} \vec{A}\right)+\frac{c}{\hbar} \vec{\Omega}\right),\left[\left(c\left(\vec{p}-\frac{e}{c} \vec{A}\right)-\frac{c}{\hbar} \vec{\Omega}\right),(\vec{E} \times \vec{p}) \cdot \vec{\Theta}\right]\right]_{-} \\
& +\frac{1}{8 m^{2} c^{4}} \frac{e}{\hbar} i \vec{\Sigma}\left\{\left[\left(c\left(\vec{p}-\frac{e}{c} \vec{A}\right)+\frac{c}{\hbar} \vec{\Omega}\right) \times\left(c\left(\vec{p}-\frac{e}{c} \vec{A}\right)-\frac{c}{\hbar} \vec{\Omega}\right),(\vec{E} \times \vec{p}) \cdot \vec{\Theta}\right]_{-},\right. \\
& \left.-2(\vec{E} \times \vec{p}) \cdot \vec{\Theta}\left(c\left(\vec{p}-\frac{e}{c} \vec{A}\right)-\frac{c}{\hbar} \vec{\Omega}\right) \times\left(c\left(\vec{p}-\frac{e}{c} \vec{A}\right)+\frac{c}{\hbar} \vec{\Omega}\right)\right\} .
\end{aligned}
$$

